\title{
Improvement of Detection Ranges for Targets in Sidelobe Clutter Surroundings by Sigma-Delta STAP for Airborne Radars
}

\author{
Tae-Hyung Kim $•$ Hyeong-Won Jeon · Jong-Hwan Shin · Yeon-Duk Kang
}

\begin{abstract}
Medium pulse repetition frequency (MPRF) waveforms are used in common airborne radar because of their advantages. However, one of the disadvantages of MPRF waveforms is the decrement of detectable ranges by sidelobe clutter at low-altitude platforms. To overcome this disadvantage and to increase the detectable ranges at low-altitude platforms, this study proposes a sidelobe clutter reduction method. Airborne radar has sum and difference antenna channels, and sidelobe clutter reduction is processed using sigma-delta spatio-temporal adaptive processing in the sidelobe-clutter surroundings of low-altitude platforms. In terms of the sidelobe clutter reduction performance and the effectiveness of increasing the detection range, the proposed method is simulated and tested using simulated data.
\end{abstract}

Key Words: Airborne Radar, MPRF Waveform, Reduction of Sidelobe Clutter, Sigma-Delta STAP, Surface Clutter.

\section{INTRODUCTION}

In the air-to-air mode of airborne pulse-Doppler radar, medium pulse repetition frequency (MPRF) waveforms can detect all aspect targets and precisely measure the range and Doppler of the targets at one time. However, despite the use of optimal PRF sets, the detection range performance of MPRF waveforms is degraded in low-altitude platforms because of the effect of sidelobe clutter $[1,2]$. In MPRF waveforms, clutter of all Doppler and ranges is folded into one PRF Doppler and one pulse repetition interval range. Folded mainlobe clutter is found in a limited Doppler region, whereas folded sidelobe clutter is found in almost all Doppler regions. In low-altitude platforms, the folded sidelobe clutter of high power affects almost all Doppler and range regions. In other words, when the platform alti- tude is low (i.e., when the power of the sidelobe clutter is high), sidelobe clutter disturbs the target detection and cause the critical degradation of the detection range performance of MPRF waveforms. Even when the detection range performance of an MPRF waveform is $80 \mathrm{~km}$ without any effects of sidelobe clutter, the performance of the MPRF waveform can be degraded to $40 \mathrm{~km}$ and below with sidelobe clutter if the platform altitude is below $2 \mathrm{~km}$. The degree of performance degradation is determined by the platform altitude and sidelobe levels of the antenna radiation pattern. Ordinary aircraft radars have sum $(\Sigma)$, diff. azimuth $(\Delta \mathrm{a})$, diff. elevation $(\Delta \mathrm{e})$, and guard $(\Lambda)$ receiving the channels of an antenna. Therefore, sigma-delta spatiotemporal adaptive processing (STAP) can be used in ordinary aircraft radars. Sigma-delta STAP was used in the mainlobe clutter reduction to detect the ground moving targets of low

Manuscript received January 31, 2019 ; Revised May 26, 2019 ; Accepted June 29, 2019. (ID No. 20190131-001J)

LIG Nex1, Yongin, Korea.

"Corresponding Author: Tae-Hyung Kim (e-mail: thkim07@gmail.com)

This is an Open-Access article distributed under the terms of the Creative Commons Attribution Non-Commercial License (http://creativecommons.org/licenses/by-nc/4.0) which permits unrestricted non-commercial use, distribution, and reproduction in any medium, provided the original work is properly cited.

(c) Copyright The Korean Institute of Electromagnetic Engineering and Science. 
velocity in the air-to-ground mode of aircraft radars [3]. This study proposes the sidelobe clutter reduction method using sigma-delta STAP. In the air-to-air mode of airborne radars that use MPRF waveforms, this sidelobe clutter reduction method improves the detection range performance, which has been degraded in the low-altitude platform. In terms of the sidelobe clutter-reduction performance and the effectiveness of increasing the detection range, the proposed method is simulated and tested using simulated data. Our method of removing sidelobe clutter is based on the same joint domain localized (JDL) method as in [3]. However, the application of algorithms for sidelobe clutter removal and the method of setting related algorithm parameters in the air-to-air environment differ from the method of removing mainlobe clutter in the air-to-ground environment of [3]. Therefore, our proposed method is also compared with the method of [3] in terms of the sidelobe-clutter-removal performance through a simulation.

\section{SIGMA-DELTA STAP FOR SIDELOBE ClutTER REDUCTION}

In this study, airborne radars have $\Sigma, \Delta \mathrm{a}, \Delta \mathrm{e}$, and $\Lambda$ receiving the channels of an antenna, and they perform the detection of air-to-air targets with these four channels. Using the sigma and delta channels to apply the STAP is an appropriate way to remove sidelobe clutter based on the STAP without configuring additional receiving channels of an antenna in the structure of antenna channels of most fighter radars. The sigma-delta STAP of this paper is similar to the JDL [4] method and is a special case of the JDL. The JDL method suppresses interference signals using the signals within the localized processing region (LPR) in the post-Doppler/beamspace domains.

A data cube, which is formed for the sigma-delta STAP, is described in Fig. 1(a). The LPR for the sigma-delta STAP in this study is illustrated in Fig. 1(b), which represents the training data collection and filter weight calculations for the STAP application to remove the sidelobe clutter. In Fig. 1(b), data $\boldsymbol{x}(n, k)$, which is processed by STAP for the elimination of the sidelobe clutter of the index $(n, k)$ point, is expressed as follows:

$$
\boldsymbol{x}(n, k)=\left[\begin{array}{c}
\boldsymbol{x}_{n-N}(k) \\
\cdots \\
\boldsymbol{x}_{n}(k) \\
\cdots \\
\boldsymbol{x}_{n+N}(k)
\end{array}\right], \quad \boldsymbol{x}_{n}(k)=\left[\begin{array}{c}
x_{\Sigma, n}(k) \\
x_{\Delta a, n}(k) \\
x_{\Delta e, n}(k)
\end{array}\right] .
$$

In $x_{c, n}(k)$ of Eq. (1), $c$ is the index of antenna receiver channel, $n$ is the range index, and $k$ is the Doppler channel index. The training data $\boldsymbol{X}$ for the STAP processing of data $\boldsymbol{x}(n, k)$ are as expressed follows:

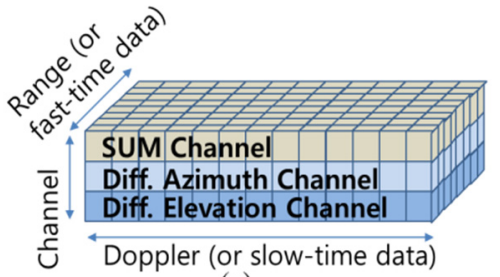

(a)

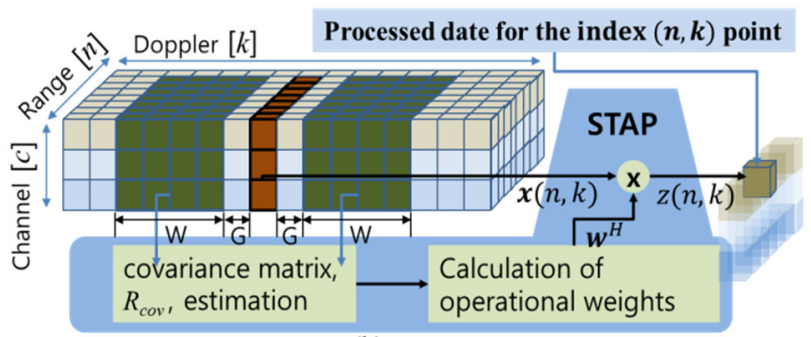

(b)

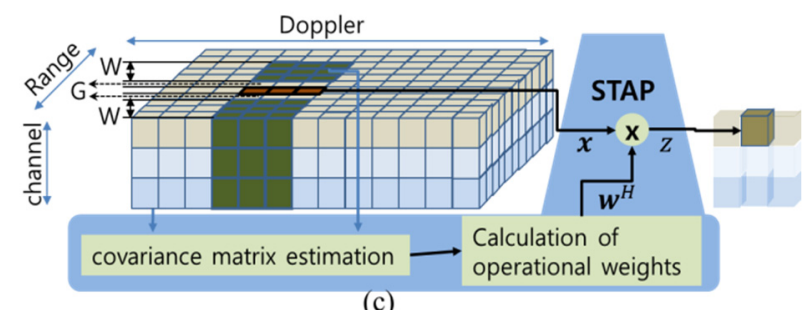

(c)

Fig. 1. (a) Data cube and (b) processing method of sigma-delta STAP for sidelobe clutter reduction, (c) processing method of sigma-delta STAP for the mainlobe clutter reduction.

$\boldsymbol{X}=[\boldsymbol{x}(n, k-A), \cdots, \boldsymbol{x}(n, k-B), \boldsymbol{x}(n, k+B), \cdots, \boldsymbol{x}(n, k+A)]$.

In Eq. (2), $A=W+G, B=G+1, W$ is the number of range cells that takes the training data around the data $\boldsymbol{x}(n, k)$, as shown in Fig. 1(b), and $G$ is the size of the guard cells that prevent the collection of training data that have information on the data $\boldsymbol{x}(n, k)$ and is set to take only the interference signal as training data. We set $N=3, W=20$, and $G=1$ in the simulation in this study. The collection of training data for the elimination of the sidelobe clutter is different from the case of eliminating the mainlobe clutter. We can compare the collections of training data of this study with the collections of training data to eliminate the mainlobe clutter in [3]. Fig. 1(c) represents the training data collection and filter weights calculations for the STAP application proposed in [3] to remove the mainlobe clutter. The main difference is that the positions of the guard cells are represented as Doppler channel indexes in the proposed method, whereas in [3], the guard cells are represented as range indexes. In other words, we collect training data on the sidelobe clutter in the Doppler direction to remove the sidelobe clutter of the $(n, k)$ position. Conversely, in [3], training data are collected in the range direction to remove the mainlobe clutter. In 
addition, there are differences not only in the direction in which the guard cell is applied but also in the direction in which the reference cell is applied and the number of reference cells. This difference in the collection of training data shows that the effect of removing the sidelobe clutter is more prominent than that of the mainlobe clutter.

The covariance matrix from the training data is expressed as follows:

$$
\boldsymbol{R}_{\text {cov }}=\frac{X X^{H}}{2 W}+\lambda \boldsymbol{I} .
$$

In Eq. (3), $\boldsymbol{X}^{H}$ is the Hermitian transpose of $X, I$ is the identity matrix, $\lambda I$ is the diagonal loading term, and $\lambda=0.01$ in our simulation ( $\lambda$ should be set appropriately according to noise level). The calculation for STAP is as follows:

$$
\boldsymbol{w}=\frac{\boldsymbol{R}_{\text {cov }}^{-1} \boldsymbol{t}}{\boldsymbol{t}^{H} \boldsymbol{R}_{\text {cov }}^{-1} \boldsymbol{t}}, \quad z(n, k)=\boldsymbol{w}^{H} \boldsymbol{x}(n, k) .
$$

In Eq. (4), $w$ is the filter weight given by the minimum variance distortionless response algorithm [3], $z(n, k)$ is the output for STAP, and $t$ is set as follows when $N=3$ :

$$
\boldsymbol{t}=[0,0,0,0,0,0,0,0,0,1,0,0,0,0,0,0,0,0,0,0,0]^{T} .
$$

\section{SiMULATION AND TEST}

In this section, we perform a simulation to test the performance of the proposed sidelobe clutter reduction method. The scenario and system parameters used in our simulation are represented in Table 1. Using the values in Table 1 and a simulated antenna pattern, we generate simulated clutter and target signals [3]. In the simulated antenna pattern of the sum channel, the power ratio of the sidelobe to the mainlobe of the antenna beam is $-60 \mathrm{~dB}$. When generating the simulated clutter signals, we use the constant gamma model as a ground clutter reflectivity model. The constant gamma item in Table 1 represents the gamma value used in the constant gamma model of this simulation. To verify the effect of increasing the detection range after removing the sidelobe clutter by our proposed method, the signal processing to detect air-to-air targets for the simulated clutter and target signals is performed, as shown in Fig. 2. In our proposed method, we set $N=3, W=20$, and $G=1$ (see Fig. 1(b)). The parameter value, which is set for the method in Fig. $1(\mathrm{c})$, is set to the value suggested in [3].

We compare the results of performing the signal processing of detecting air-to-air targets without removing the sidelobe clutter. The results of removing the sidelobe clutter using the method in [3] (Fig. 1(c)) are compared with those of removing the sidelobe clutter by the method presented in this paper. Fig. 3 compares the powers of the clutter of the range/Doppler (RD) maps with the results of Doppler processing before and after

\begin{tabular}{|c|c|c|c|}
\hline \multicolumn{4}{|c|}{ Clutter and target signal } \\
\hline I & & & $T$ \\
\hline SUM ch. & Diff. Az. ch. & Diff. El. ch. & Guard ch. \\
\hline 1 & & & $\downarrow$ \\
\hline \multicolumn{4}{|c|}{ Pulse compression } \\
\hline I & 1 & $\downarrow$ & I \\
\hline \multicolumn{4}{|c|}{ Doppler processing } \\
\hline$I$ & & 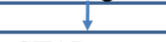 & \\
\hline \multicolumn{3}{|c|}{ Datacube formation for STAP } & \\
\hline \multirow{2}{*}{\multicolumn{3}{|c|}{ STAP processing }} & \\
\hline & & & \\
\hline & \\
\hline & \multicolumn{2}{|c|}{ CFAR processing } & \\
\hline \multicolumn{3}{|c|}{ SLB processing } & \\
\hline ext & $\begin{array}{r}\text { Hit clu } \\
\text { ting informatic }\end{array}$ & $\begin{array}{l}\text { stering and } \\
\text { n of detectec }\end{array}$ & gets \\
\hline
\end{tabular}

Table 1. Scenario and system parameters in this simulation

\begin{tabular}{lc}
\multicolumn{1}{c}{ Parameter } & Value \\
\hline Platform altitude $(\mathrm{km})$ & 3 \\
Platform velocity $(\mathrm{m} / \mathrm{s})$ & 200 \\
One target, range $(\mathrm{km})$ & 73 \\
One target, RCS $\left(\mathrm{m}^{2}\right)$ & 1 \\
Constant gamma & 0.06 \\
Transmission frequency $(\mathrm{GHz})$ & 10 \\
PRF $(\mathrm{kHz})$ & 20 \\
Transmission pulse width $(\mu \mathrm{s})$ & 8 \\
Sampling frequency $(\mathrm{MHz})$ & 5 \\
CFAR threshold $($ false alarm rate $)$ & $10^{-6}$ \\
\hline
\end{tabular}

Fig. 2. Signal processing for target detection.

removing the sidelobe clutter. Fig. 3(a) shows the clutter before removing the sidelobe clutter, and Fig. 3(b) illustrates the clutter after removing the sidelobe clutter using the proposed method. Fig. 3(c) shows the clutter after removing the sidelobe clutter using the method in [3] (Fig. 1(c)). The dotted square areas shown in each RD map of Fig. 3 are enlarged and displayed at the bottom of each RD map. Before removing the sidelobe clutter, a target is buried in the clutter in the dotted square area, and the target is invisible (Fig. 3(a)). After removing the sidelobe clutter using the proposed method, the target appears in the area (Fig. 3(b)) and has a detectable signal-to-interference and noise ratio (SINR). Note that the detection performance of the CFAR processing in Fig. 2 is determined by the SINR around the targets. In Fig. 3(a), the signal-to-noise ratio (SNR) of the target is $16.4 \mathrm{~dB}$, the clutter-to-noise ratio (CNR) around the target is $12.55 \mathrm{~dB}$, and the SINR around the target is $3.85 \mathrm{~dB}$. In Fig. 3(b), SNR of the target is $15.2 \mathrm{~dB}$, the CNR around the target is $0.31 \mathrm{~dB}$, and the SINR around the target is $14.89 \mathrm{~dB}$. In Fig. 3(c), SNR of the target is $15.95 \mathrm{~dB}, \mathrm{CNR}$ around the target is about $11.03 \mathrm{~dB}$, and SINR around the target is about $4.92 \mathrm{~dB}$. Fig. 3(a) and (b) show the $11.04 \mathrm{~dB}(=14.89-3.85 \mathrm{~dB})$ 


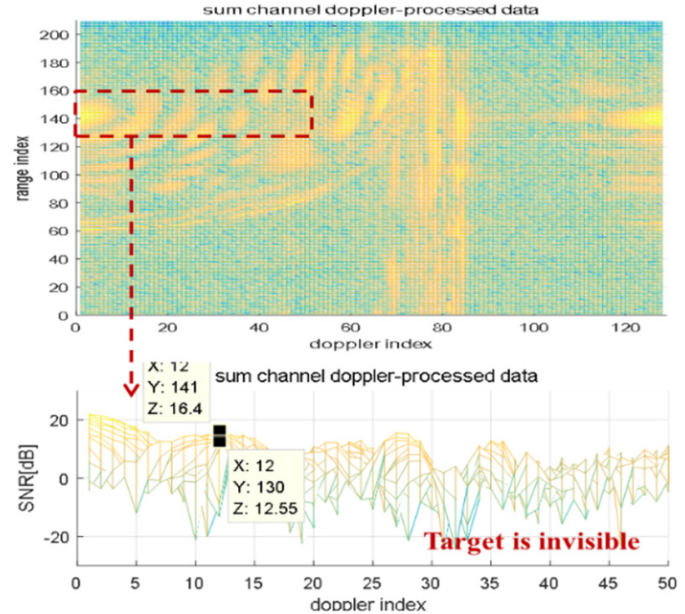

(a)

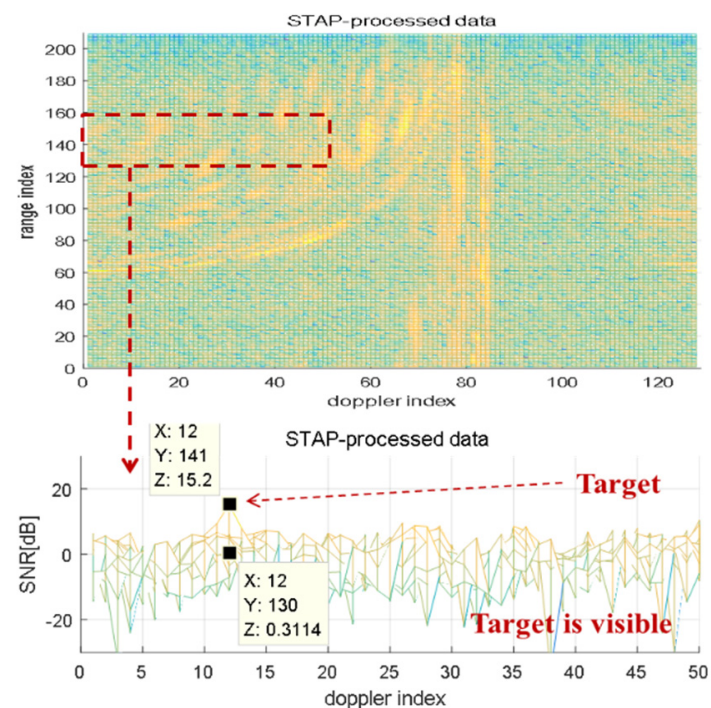

(b)

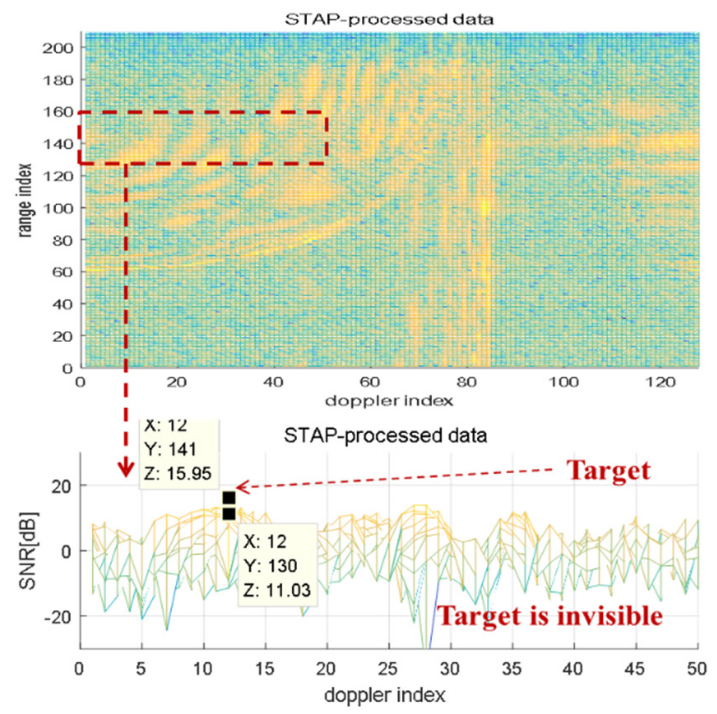

(c)

Fig. 3. RD maps before and after removing the sidelobe clutter: (a) before removing sidelobe cutter, (b) after removing the sidelobe cutter using the proposed method in Fig. 1(b), and (c) after removing the sidelobe cutter using the method in Fig. 1(c).
SINR improvement using the proposed method. The CNR of the clutter around the target decreases to $0.31 \mathrm{~dB}$ from 12.55 $\mathrm{dB}$ after the sidelobe clutter is removed (sidelobe clutter reduction performance is $12 \mathrm{~dB}$ or more). Conversely, Fig. 3(a) and (c) show only about $1.07 \mathrm{~dB}(=4.92-3.85 \mathrm{~dB})$ of SINR improvement using the method in [3], and CNR around the target decreases to only $11.03 \mathrm{~dB}$ from $12.55 \mathrm{~dB}$. After removing the sidelobe clutter using the method in [3], the target remains buried in the clutter in the dotted square area, thus making it invisible (Fig. 3(c)). As shown in Fig. 3, the sidelobe clutter is not properly removed by the method in [3] and sufficient SINR is not secured.

Fig. 4(a) shows the hit clustering result of the signal processing of detecting targets without removing the sidelobe clutter. Fig. 4(b) presents the result of detecting targets with removing the sidelobe clutter using the proposed method. Fig. 4(c) shows the result of detecting targets with removing the sidelobe clutter using the method in [3].

The target range of $73 \mathrm{~km}$, which is not detected because

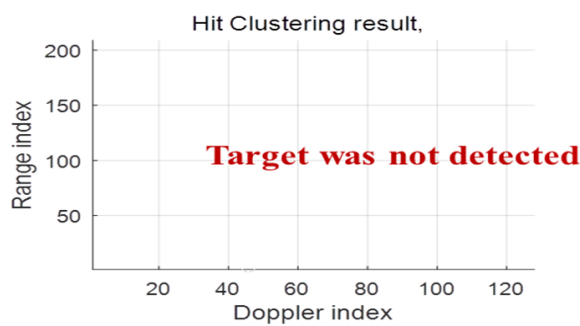

(a)

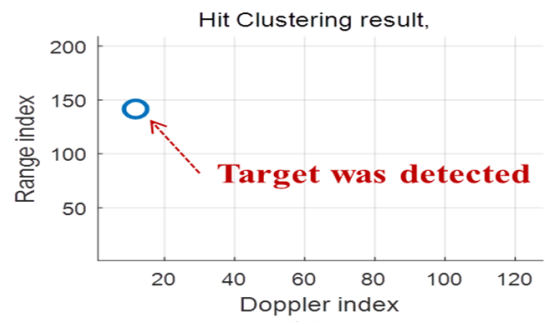

(b)

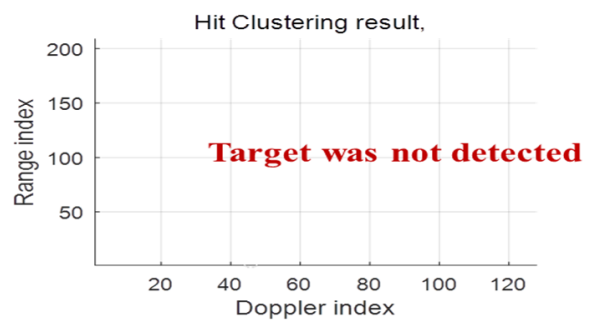

(c)

Fig. 4. Target detection results: (a) without removing the sidelobe clutter, the target is not detected [Fig. 3(a)], (b) the target was detected using the proposed method [Fig. 3(b)], and (c) the target was not detected [Fig. 3(c)] by removing the sidelobe clutter using the method in [3]. 
of the sidelobe clutter in the platform altitude of $3 \mathrm{~km}$, is detected by removing the sidelobe clutter using the proposed method.

\section{CONCLUSION}

To counter the degradation of the detection-range performance of air-to-air targets due to sidelobe clutter at low altitudes of airborne radars in MPRF waveforms, we suggested a method of removing the sidelobe clutter using sigma-delta STAP. The sidelobe clutter reduction performance of the proposed method was verified at $12 \mathrm{~dB}$ through simulations. The effectiveness of increasing the detection range was verified through signal processing simulations that detect targets.

\section{Tae-Hyung Kim}

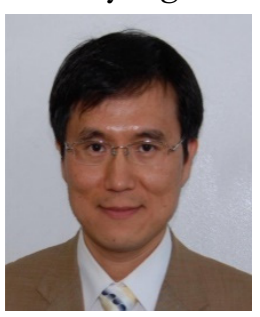

received his $\mathrm{Ph} . \mathrm{D}$. degree in electronic engineering from Pusan National University, Busan, Korea, in 2007. From 2007 to 2010, he worked at the Agency for Defense Development, Daejeon, Korea. He is currently a researcher at LIG Nex1. His research interests include radar signal processing, modelling, and simulation for radar systems and pattern recognition.

Hyeong-Won Jeon

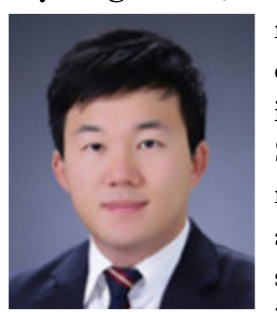

received his B.S. and M.S. degrees in information communication engineering from Hanbat University in Daejeon, Korea, in 2010 and Gwangju Institute of Science and Technology in Gwangju, Korea, in 2012, respectively. He is currently working at LIG Nex1 as a researcher. His research interests include radar signal processing and embedded real-time programing.

\section{REFERENCES}

[1] M. I. Skolnik, Radar Handbook, 3rd ed. New York, NY: McGraw-Hill, 2008.

[2] T. H. Kim, J. W. Yi, and Y. J. Byun, "Real-time selection of pulse repetition frequency (PRF) set for a triple 2-of-3 PRF scheme," Journal of Electromagnetic Engineering and Science, vol. 13, no. 3, pp. 186-188, 2013.

[3] T. H. Kim, J. S. Yoon, J. H. Jung, and S. H. Ryu, "Application design and performance analysis simulation of sigmadelta STAP for GMTI mode of airborne radar," The Journal of Korean Institute of Electromagnetic Engineering and Science, vol. 28, no. 4, pp. 336-346, 2017.

[4] H. Wang and L. Cai, "A localized adaptive MTD processor," IEEE Transactions on Aerospace and Electronic Systems, vol. 27, no. 3, pp. 532-539, 1991.

\section{Jong-Hwan Shin}

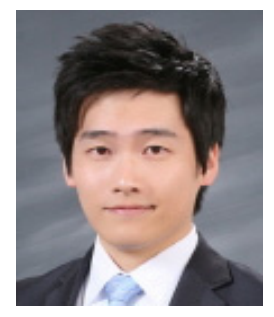

received his B.S. degree in electronic and computer science engineering from Inha University in Incheon, Korea, in 2012. He is currently working at LIG Nex1 as a researcher. His research interests include radar system control, communication, and artificial intelligence. received his B.S. and M.S. degrees in electrical engineering from Korea Aerospace University in Korea in 1993 and 1996, respectively, and his Ph.D. degree in electrical engineering from Korea Aerospace University in Korea in 2000. Since 2000, he has been working at LIG Nex1 as a researcher. His current research interests include phased array antennas and radar systems. 